Article

\title{
The Optimal Fix-Free Code for Anti-Uniform Sources
}

\author{
Ali Zaghian ${ }^{1}$, Adel Aghajan ${ }^{2}$ and T. Aaron Gulliver ${ }^{3, *}$ \\ ${ }^{1}$ Department of Mathematics and Cryptography, Malek Ashtar University of Technology, Isfahan \\ 83145/115, Iran; E-Mail: a_zaghian@mut-es.ac.ir \\ ${ }^{2}$ Department of Electrical and Computer Engineering, Isfahan University of Technology, Isfahan \\ 84156-83111, Iran; E-Mail: a.aghajanabdollah@ec.iut.ac.ir \\ ${ }^{3}$ Department of Electrical and Computer Engineering, University of Victoria, PO Box 1700, STN CSC, \\ Victoria, BC V8W 2Y2, Canada \\ * Author to whom correspondence should be addressed; E-Mail: agullive@ece.uvic.ca; \\ Tel.: +1-250-721-6028; Fax: +1-250-721-6052.
}

Academic Editor: Raúl Alcaraz Martínez

Received: 9 February 2015 / Accepted: 13 March 2015 / Published: 19 March 2015

\begin{abstract}
An $n$ symbol source which has a Huffman code with codelength vector $L_{n}=(1,2,3, \cdots, n-2, n-1, n-1)$ is called an anti-uniform source. In this paper, it is shown that for this class of sources, the optimal fix-free code and symmetric fix-free code is $C_{n}^{*}=(0,11,101,1001, \cdots, 1 \overbrace{0 \cdots 0}^{n-2} 1)$.
\end{abstract}

Keywords: prefix code; fix-free code; anti-uniform sources

\section{Introduction}

One of the basic problems in the context of source coding is to assign a code $C_{n}=\left(c_{1}, c_{2}, \cdots, c_{n}\right)$ with codelength vector $L_{n}=\left(\ell_{1}, \ell_{2}, \cdots, \ell_{n}\right)$ to a memoryless source with probability vector $P_{n}=\left(p_{1}, p_{2}, \cdots, p_{n}\right)$. Decoding requirements often constrain us to choose a code $C_{n}$ from a specific class of codes, such as prefix-free codes, fix-free codes or symmetric fix-free codes. With a prefix-free code, no codeword is the prefix of another codeword. This property ensures that decoding in the forward direction can be done without any delay (instantaneously). Alternatively, with a fix-free code no codeword is the prefix or suffix of any other codeword [1,2]. Therefore, decoding of a fix-free code 
in both the forward and backward directions can be done without any delay. The ability to decode a fix-free code in both directions makes them more robust to transmission errors and faster decoding can be achieved compared to prefix-free codes. As a result, fix-free codes are used in video standards such as H.263+ and MPEG-4 [3]. A symmetric fix-free code is a fix-free code whose codewords are symmetric. In general, decoder implementation for a fix-free code requires more memory compared to that for a prefix-free code. Although the decoder for a symmetric fix-free code is the same as for a fix-free code [4], symmetric codes have greater redundancy in general.

Let $S\left(L_{n}\right)=\sum_{i=1}^{n} 2^{-\ell_{i}}$ denote the Kraft sum of the codelength vector $L_{n}$. A well-known necessary and sufficient condition for the existence of a prefix-free code with codelength vector $L_{n}$ is the Kraft inequality, i.e., $S\left(L_{n}\right) \leq 1$ [5]. However, this inequality is only a necessary condition on the existence of a fix-free code. Some sufficient conditions on the existence of a fix-free code were introduced in [6-11].

The optimal code for a specific class of codes is defined as the code with the minimum average codelength, i.e., $\sum_{i=1}^{n} p_{i} \ell_{i}$ among all codes in that class. The optimal prefix-free code can easily be obtained using the Huffman algorithm [12]. Recently, two methods for finding the optimal fix-free code have been developed. One is based on the $A^{*}$ algorithm [13], while the other is based on the concept of dominant sequences [14]. Compared to the Huffman algorithm, these methods are very complex.

A source with $n$ symbols having Huffman code with codelength vector $L_{n}=(1,2,3, \cdots, n-2, n-$ $1, n-1)$ is called an anti-uniform source $[15,16]$. Such sources have been shown to correspond to particular probability distributions. For example, it was shown in [17] and [18], respectively, that the normalized tail of the Poisson distribution and the geometric distribution with success probability greater than some critical value are anti-uniform sources. It was demonstrated in $[15,16]$ that a source with probability vector $P_{n}=\left(p_{1}, p_{2}, \cdots, p_{n}\right)$ where $p_{1} \geq p_{2} \geq \cdots \geq p_{n}$, is anti-uniform if and only if

$$
\sum_{j=i+2}^{n} p_{j} \leq p_{i} \quad \text { for } \quad 1 \leq i \leq n-3 .
$$

As mentioned above, finding an optimal fix-free or symmetric fix-free code is complex. Thus, in this paper optimal fix-free and symmetric fix-free codes are determined for anti-uniform sources. In particular, it is proven that

$$
C_{n}^{*}=(0,11,101,1001, \cdots, 1 \overbrace{0 \cdots 0}^{n-2} 1),
$$

is an optimal fix-free code for this class of sources. Since $C_{n}^{*}$ is symmetric, this code is also an optimal symmetric fix-free code. Although for an anti-uniform source, the difference between the average codelength of the optimal prefix-free code and $C_{n}^{*}$ is small (it is exactly equal to $p_{n}$ ), it is not straightforward to prove that $C_{n}^{*}$ is the optimal fix-free code. In [19], the optimality of $C_{n}^{*}$ among symmetric fix-free codes for a family of exponential probability distributions, which is an anti-uniform source, was discussed.

In [20], another class of fix-free codes called weakly symmetric fix-free codes was examined. A fix-free code is weakly-symmetric if the reverse of each codeword is also a codeword. In fact, every symmetric fix-free code is a weakly symmetric fix-free code, and every weakly symmetric fix-free code is a fix-free code. Thus, since the optimal code among fix-free codes and symmetric fix-free codes for anti-uniform sources is $C_{n}^{*}$, this code is also optimal for weakly symmetric fix-free codes. 
The remainder of this paper is organized as follows. In Section 2, a sketch of the proofs of the main theorems, i.e., Theorems 1 and 3, is provided, followed by the main results of the paper. Then detailed proofs of these results are given in Section 3.

\section{A Sketch of the Proofs}

Since a fix-free code is also a prefix-free code, the Kraft sum of an optimal fix-free code is not greater than 1 . Therefore, the Kraft sum of this code is either equal to 1 or smaller than 1.

Proposition 1. If

$$
\left(\ell_{1}^{*}, \cdots, \ell_{n-1}^{*}, \ell_{n}^{*}\right)=\arg \min _{L_{n}: S\left(L_{n}\right) \leq 1} \sum_{i=1}^{n} p_{i} \ell_{i},
$$

then we have

$$
\left(\ell_{1}^{*}, \cdots, \ell_{n-1}^{*}, \ell_{n}^{*}+1\right)=\arg \min _{L_{n}: S\left(L_{n}\right)<1} \sum_{i=1}^{n} p_{i} \ell_{i} .
$$

It can be inferred from Proposition 1 that if the Kraft sum of an optimal fix-free code is smaller than 1 , then the average codelength of this code is not better than the codelength vector $\left(\ell_{1}^{*}, \cdots, \ell_{n-1}^{*}, \ell_{n}^{*}+1\right)$. The optimal prefix-free code for an anti-uniform source has codelength vector $(1,2, \cdots, n-1, n-1)$. Therefore, the optimal codelength vector with Kraft sum smaller than 1 for an anti-uniform source is the codelength vector $(1,2, \cdots, n-1, n)$. Further, the codelength vector of $C_{n}^{*}$ is $(1,2, \cdots, n-1, n)$. Thus, if the Kraft sum of the optimal fix-free code for an anti-uniform source is smaller than 1 , then the code $C_{n}^{*}$ is optimal.

Proposition 2. There is no symmetric fix-free code with Kraft sum 1 for $n>2$.

According to Proposition 2, the Kraft sum for an optimal symmetric fix-free code is smaller than 1. Thus, Propositions 1 and 2 prove the following theorem.

Theorem 1. The optimal symmetric fix-free code for an anti-uniform source $P_{n}$ is the code $C_{n}^{*}$.

There exist fix-free codes with Kraft sum 1, for example $(00,01,10,11)$ and $(01,000,100,110,111,0010,0011,1010,1011)$ [21]. Therefore, proving that the code $C_{n}^{*}$ is the optimal fix-free code for anti-uniform sources requires that the average codelength for this code be better than every possible codelength for a fix-free code. To achieve this, we use the following theorem which was proven in [21].

Theorem 2. [21] Let $L_{n}=\left(\ell_{1}, \cdots, \ell_{n}\right), M_{i}\left(L_{n}\right)=\left|\left\{j \mid \ell_{j}=i\right\}\right|$ for $1 \leq i \leq \max _{1 \leq j \leq n} \ell_{j}$ and $H_{i}=2 i-\frac{1}{i} \sum_{j=1}^{i} 2^{(i, j)}$ where $(i, j)$ denotes the greatest common divisor of $i$ and $j$. If $S\left(L_{n}\right)=1$, $M_{i}\left(L_{n}\right)>H_{i}$ for some $i$ and $\left|\left\{\ell_{1}, \cdots, \ell_{n}\right\}\right|>1$, then no fix-free code exists with codelength vector $L_{n}$.

According to the definition of $H_{i}$, we have that $H_{1}=0$ and $H_{2}=1$. Therefore from Theorem 2, for $L_{n}$ with Kraft sum 1 and

$$
M_{1}\left(L_{n}\right)>0 \text { and } L_{n} \neq(1,1) \text {, }
$$


or

$$
M_{2}\left(L_{n}\right)>1 \text { and } L_{n} \neq(2,2,2,2)
$$

there is no fix-free code.

Definition 1. For a given n, let

$$
\mathbb{L}_{n}=\left\{L_{n} \mid S\left(L_{n}\right)=1, M_{1}\left(L_{n}\right)=0 \text { and } M_{2}\left(L_{n}\right) \leq 1\right\} .
$$

From Theorem 2, if the Kraft sum of the optimal fix-free code is equal to 1, then the average codelength for this code is not smaller than that of the optimal codelength vector among those in $\mathbb{L}_{n}$ for $n>4$. It can easily be verified that $\left|\mathbb{L}_{n}\right|=0$ for $n<7$. For anti-uniform sources, the following proposition characterizes the optimal codelength vector in $\mathbb{L}_{n}$ for $n \geq 7$.

Proposition 3. Let $P_{n}=\left(p_{1}, \cdots, p_{n-1}, p_{n}\right)$ be the probability vector of an anti-uniform source with $p_{1} \geq \cdots \geq p_{n-1} \geq p_{n}$. Then we have

$$
\arg \min _{L_{n} \in \mathbb{L}_{n}} \sum_{i=1}^{n} p_{i} \ell_{i}= \begin{cases}(2,3,3,3,3,3,3), & \text { if } n=7 \\ (2,3,3,3,3,3,4,5,6, \cdots, n-6, n-5, n-4, n-4), & \text { if } n>7\end{cases}
$$

The last step requires that the average codelength of $C_{n}^{*}$ is better than that of the given codelength vector in Proposition 3. This is given in the proof of the following theorem.

Theorem 3. The optimal fix-free code for an anti-uniform source $P_{n}$ is $C_{n}^{*}$ for $n>4$.

Note that Theorem 3 is not true for $n=4$. For example, for $P_{4}=\left(\frac{1}{3}, \frac{1}{3}, \frac{1}{6}, \frac{1}{6}\right)$ which is the probability vector of an anti-uniform source, the average codelength of the fix-free code $(00,01,10,11)$ is better than that of $C_{4}^{*}$.

\section{Proofs of the Results in Section 2}

Proof of Proposition 1: Let $L_{n}=\left(\ell_{1}, \cdots, \ell_{n-1}, \ell_{n}\right)$ with $\ell_{1} \leq \cdots \leq \ell_{n-1} \leq \ell_{n}$ and $1>S\left(L_{n}\right)$. Thus, we have $2^{\ell_{n}}>2^{\ell_{n}} S\left(L_{n}\right)=\sum_{i=1}^{n} 2^{\ell_{n}-\ell_{i}}$, and consequently

$$
2^{\ell_{n}} \geq 1+\sum_{i=1}^{n} 2^{\ell_{n}-\ell_{i}} .
$$

Therefore, we can write

$$
\begin{aligned}
1 & \geq \sum_{i=1}^{n} 2^{-\ell_{i}}+2^{-\ell_{n}} \\
& =\sum_{i=1}^{n-1} 2^{-\ell_{i}}+2^{-\left(\ell_{n}-1\right)} .
\end{aligned}
$$

Let $L_{n}^{\prime}=\left(\ell_{1}^{\prime}, \cdots, \ell_{n-1}^{\prime}, \ell_{n}^{\prime}\right)$ such that $\ell_{i}^{\prime}=\ell_{i}$ for $1 \leq i \leq n-1$ and $\ell_{n}^{\prime}=\ell_{n}-1$. From (2), we have that $S\left(L_{n}^{\prime}\right) \leq 1$. According to the definition of $\left(\ell_{1}^{*}, \cdots, \ell_{n-1}^{*}, \ell_{n}^{*}\right)$, we can write

$$
\sum_{i=1}^{n} p_{i} \ell_{i}^{*} \leq \sum_{i=1}^{n} p_{i} \ell_{i}^{\prime}
$$


and consequently

$$
\begin{aligned}
\sum_{i=1}^{n-1} p_{i} \ell_{i}^{*}+\left(\ell_{n}^{*}+1\right) p_{n} & =\sum_{i=1}^{n} p_{i} \ell_{i}^{*}+p_{n} \\
& \leq \sum_{i=1}^{n} p_{i} \ell_{i}^{\prime}+p_{n} \\
& =\sum_{i=1}^{n} p_{i} \ell_{i} .
\end{aligned}
$$

This shows that the average codelength of $\left(\ell_{1}^{*}, \cdots, \ell_{n-1}^{*}, \ell_{n}^{*}+1\right)$ is better than any other codelength vector, say $L_{n}$, with Kraft sum smaller than 1.

Proof of Proposition 2: Suppose that the Kraft sum of $L_{n}$, which is the codelength vector of the code $C_{n}$, is equal to 1 . Let codeword $c=x_{1} x_{2} \cdots x_{\ell-1} 0$ (resp. $c=x_{1} x_{2} \cdots x_{\ell-1} 1$ ) with length $\ell(\ell>1)$, be the longest codeword of $C_{n}$. Since the Kraft sum of $L_{n}$ is equal to 1 , the codeword $c^{\prime}=x_{1} x_{2} \cdots x_{\ell-1} 1$ (resp. $c^{\prime}=x_{1} x_{2} \cdots x_{\ell-1} 0$ ) belongs to $C_{n}$. However, both $c$ and $c^{\prime}$ cannot be symmetric because $x_{1}=0$ and $x_{1}=1$ cannot both be true. Thus, $C_{n}$ is not a symmetric fix-free code.

The following lemma will be used in the proof of Proposition 3.

Lemma 1. For $n \geq 7$, let $P_{n}=\left(p_{1}, \cdots, p_{n-1}, p_{n}\right)$ with $p_{1} \geq \cdots \geq p_{n-1} \geq p_{n}$ and

$$
P_{n-1}^{\prime}=\left(p_{1}^{\prime}, \cdots, p_{n-2}^{\prime}, p_{n-1}^{\prime}\right)=\left(p_{1}, \cdots, p_{n-2}, p_{n-1}+p_{n}\right)
$$

Further, suppose that

$$
L_{n}^{*}=\left(\ell_{1}^{*}, \cdots, \ell_{n-1}^{*}, \ell_{n}^{*}\right)=\arg \min _{L_{n} \in \mathbb{L}_{n}} \sum_{i=1}^{n} p_{i} \ell_{i},
$$

and for $n>7$

$$
L_{n-1}^{\prime}=\left(\ell_{1}^{\prime}, \cdots, \ell_{n-2}^{\prime}, \ell_{n-1}^{\prime}\right)=\arg \min _{L_{n-1} \in \mathbb{L}_{n-1}} \sum_{i=1}^{n-1} p_{i}^{\prime} \ell_{i}
$$

Then we have

$$
\left(\ell_{1}^{*}, \cdots, \ell_{n-2}^{*}, \ell_{n-1}^{*}, \ell_{n}^{*}\right)= \begin{cases}(2,3,3,3,3,3,3), & \text { if } n=7 \\ \left(\ell_{1}^{\prime}, \cdots, \ell_{n-2}^{\prime}, \ell_{n-1}^{\prime}+1, \ell_{n-1}^{\prime}+1\right), & \text { if } n>7\end{cases}
$$

Proof. It can easily be verified that $\mathbb{L}_{7}$ consists of all permutations of $2,3,3,3,3,3,3$. Thus, $p_{1} \geq \cdots \geq$ $p_{6} \geq p_{7}$ completes the proof for $n=7$. To prove the lemma for $n>7$, we consider two cases: (1) $\ell_{n}^{*}=3$, and (2) $\ell_{n}^{*}>3$. First, note that $\ell_{n}^{*}=\max _{1 \leq i \leq n} \ell_{i}$, because $p_{1} \geq \cdots \geq p_{n-1} \geq p_{n}$.

(1) $\ell_{n}^{*}=3$ : It can easily be shown that $(3,3,3,3,3,3,3,3)$ is the only codelength vector in $\cup_{n>7} \mathbb{L}_{n}$ with maximum codelength which is a not greater than 3 . Therefore, to prove the lemma in this case it is enough to show that $\left(\ell_{1}^{\prime}, \cdots, \ell_{7}^{\prime}\right)=(3,3,3,3,3,3,2)$. According to the first argument in this proof, the codelength vector $\left(\ell_{1}^{\prime}, \cdots, \ell_{7}^{\prime}\right)$ is a permutation of $2,3,3,3,3,3,3$. Thus, proving that $p_{7}+p_{8}$ is maximum over all probabilities in $P_{7}^{\prime}$, i.e., $p_{7}+p_{8} \geq p_{1}$, completes the proof for this case. If 
$p_{7}+p_{8}<p_{1}$, then the average codelength of $\left(\ell_{1}^{\prime \prime}, \cdots, \ell_{8}^{\prime \prime}\right)=(2,3,3,3,3,3,4,4)$, is better than that of $\left(\ell_{1}^{*}, \cdots, \ell_{8}^{*}\right)=(3,3,3,3,3,3,3,3)$, because

$$
\begin{aligned}
\sum_{i=1}^{8} p_{i} \ell_{i}^{*} & =3 p_{1}+3\left(1-p_{1}\right) \\
& \stackrel{(\text { a) }}{>} 2 p_{1}+3\left(1-p_{1}\right)+p_{7}+p_{8} \\
& =\sum_{i=1}^{8} p_{i} \ell_{i}^{\prime \prime},
\end{aligned}
$$

where (a) follows from $p_{7}+p_{8}<p_{1}$. Thus, the codelength vector $(3,3,3,3,3,3,3)$ is not optimal, which is a contradiction. Therefore, $p_{7}+p_{8} \geq p_{1}$ and the proof for this case is complete.

(2) $\ell_{n}^{*}>3$ : The proof for this case is similar to the proof of the Huffman algorithm. Let $L_{n}=$ $\left(\ell_{1}, \cdots, \ell_{n}\right) \in \mathbb{L}_{n}$, i.e., $S\left(L_{n}\right)=1, M_{1}\left(L_{n}\right)=0$ and $M_{2}\left(L_{n}\right) \leq 1$, with $\ell_{1} \leq \cdots \leq \ell_{n}$ and $3<\ell_{n}$, and let $L_{n-1}^{\prime \prime}=\left(\ell_{1}^{\prime \prime}, \cdots, \ell_{n-1}^{\prime \prime}\right)=\left(\ell_{1}, \cdots, \ell_{n-2}, \ell_{n-1}-1\right)$. It can easily be shown that $S\left(L_{n}\right)=1$ implies that $\ell_{n}=\ell_{n-1}$. Since $\ell_{n}=\ell_{n-1}$, we have $S\left(L_{n-1}^{\prime \prime}\right)=S\left(L_{n}\right)$, and consequently $S\left(L_{n-1}^{\prime \prime}\right)=1$. Further, since $\ell_{n}=\max _{1 \leq i \leq n} \ell_{i}$ and $\ell_{n}>3, M_{1}\left(L_{n}\right)=0$ and $M_{2}\left(L_{n}\right) \leq 1$ imply that $M_{1}\left(L_{n-1}^{\prime \prime}\right)=0$ and $M_{2}\left(L_{n-1}^{\prime \prime}\right) \leq 1$, which gives $L_{n-1}^{\prime \prime} \in \mathbb{L}_{n-1}$, and we can write

$$
\begin{aligned}
\sum_{i=1}^{n} p_{i} \ell_{i}^{*} & =\min _{L_{n} \in \mathbb{L}_{n}} \sum_{i=1}^{n} p_{i} \ell_{i} \\
& \stackrel{(\mathrm{a})}{=}\left[\min _{L_{n} \in \mathbb{L}_{n}} \sum_{i=1}^{n-2} p_{i} \ell_{i}+\left(p_{n-1}+p_{n}\right)\left(\ell_{n-1}-1\right)\right]+p_{n-1}+p_{n} \\
& \stackrel{(\mathrm{b})}{=}\left[\min _{L_{n-1}^{\prime \prime} \in \mathbb{L}_{n-1}} \sum_{i=1}^{n-1} p_{i}^{\prime} \ell_{i}^{\prime \prime}\right]+p_{n-1}+p_{n} \\
& \stackrel{(\mathrm{c})}{=} \sum_{i=1}^{n-1} p_{i}^{\prime} \ell_{i}^{\prime}+p_{n-1}+p_{n},
\end{aligned}
$$

where (a) follows from $\ell_{n}^{*}=\ell_{n-1}^{*}$, (b) follows from the definition of $L_{n-1}^{\prime \prime}$ and (3), and (c) follows from (4). Therefore, for $n>7$, since the average codelength of the given codelength vector in (5) is equal to $\sum_{i=1}^{n-1} p_{i}^{\prime} \ell_{i}^{\prime}+p_{n-1}+p_{n}$, this codelength vector is optimal and the proof is complete.

Proof of Proposition 3. The proposition is proved by induction on $n$. According to Lemma 1, the base of induction, i.e., $n=7$, is true. Assume that the proposition is true for all anti-uniform sources with $n-1$ symbols. Let $P_{n}=\left(p_{1}, \cdots, p_{n}\right)$ be the probability vector of an anti-uniform source. Also, suppose that $P_{n-1}^{\prime}=\left(p_{1}^{\prime}, \cdots, p_{n-2}^{\prime}, p_{n-1}^{\prime}\right)=\left(p_{1}, \cdots, p_{n-2}, p_{n-1}+p_{n}\right)$. From (1), it is obvious that $P_{n-1}^{\prime}$ is the probability vector of an anti-uniform source and $p_{1}^{\prime} \geq p_{2}^{\prime} \geq \cdots \geq p_{n-3}^{\prime} \geq p_{n-2}^{\prime}, p_{n-1}^{\prime}$. Since we have $\ell_{n-1}^{\prime}=\ell_{n}^{\prime}$, where $\left(\ell_{1}^{\prime}, \cdots, \ell_{n-2}^{\prime}, \ell_{n-1}^{\prime}\right)=\arg \min _{L_{n-1} \in \mathbb{L}_{n-1}} \sum_{i=1}^{n-1} p_{i}^{\prime} \ell_{i}$, from the induction assumption we can write

$$
\left(\ell_{1}^{\prime}, \cdots, \ell_{n-2}^{\prime}, \ell_{n-1}^{\prime}\right)=\left\{\begin{array}{ll}
(2,3,3,3,3,3,3), & \text { if } n-1=7 \\
(2,3,3,3,3,3,4,5, \cdots, n-5, n-5), & \text { if } n-1>7
\end{array} .\right.
$$


Therefore, we have $\ell_{n-1}^{\prime}=n-5$, and Lemma 1 completes the proof.

Proof of Theorem 3. We have that $\left|\mathbb{L}_{5}\right|=\left|\mathbb{L}_{6}\right|=0$. Therefore, for $n=5,6$ the proof is complete. The proof for $n=7$ is the same as the proof for $n>7$, and so is omitted. Now suppose that $n>7$. In the following, it is proven that the average codelength vector of $\left(\ell_{1}^{\prime}, \ldots, \ell_{n}^{\prime}\right)=$ $(2,3,3,3,3,3,4,5,6, \cdots, n-4, n-4)$ is greater than or equal to that of $C_{n}^{*}$, i.e., $\sum_{i=1}^{n} i p_{i}$, which completes the proof.

$$
\begin{aligned}
\sum_{i=1}^{n} p_{i} \ell_{i}^{\prime} & =2 p_{1}+3\left(p_{2}+p_{3}+p_{4}+p_{5}+p_{6}\right)+\sum_{i=7}^{n-1}(i-3) p_{i}+(n-4) p_{n} \\
& =\sum_{i=1}^{n} i p_{i}+p_{1}+p_{2}-p_{4}-2 p_{5}-3 p_{6}-3 \sum_{i=7}^{n} p_{i}-p_{n} \\
& =\sum_{i=1}^{n} i p_{i}+\left(p_{1}-p_{3}-p_{4}-p_{n}\right)+\left(p_{2}-\sum_{i=4}^{n} p_{i}\right)+\left(p_{3}-\sum_{i=5}^{n} p_{i}\right)+\left(p_{4}-\sum_{i=6}^{n} p_{i}\right) \\
& \stackrel{\text { (a) }}{\geq} \sum_{i=1}^{n} i p_{i},
\end{aligned}
$$

where (a) follows from the fact that $P_{n}$ is the probability vector of an anti-uniform source, i.e., $p_{i} \geq$ $\sum_{j=i+2}^{n} p_{j}$ for $n-3 \geq i \geq 1$.

\section{Author Contributions}

All authors were involved in developing the problem, interpreting the results, and writing the paper. Ali Zaghian and Adel Aghajan conducted the analysis. All authors have read and approved the final manuscript.

\section{Conflicts of Interest}

The authors declare no conflict of interest.

\section{References}

1. Schützenberg, M.P. On an application of semigroup methods to some problems in coding. IRE Trans. Inf. Theory 1956, 2, 47-60.

2. Gilbert, E.N.; Moore, E.F. Variable-length binary encodings. Bell Syst. Tech. J. 1959, 38, 933-968.

3. Webb, J.L.H. Efficient table access for reversible variable-length decoding. IEEE Trans. Circuits Syst. Video Technol. 2001, 11, 981-985.

4. Takishima, Y; Wada, M.; Murakami, H. Reversible variable length codes. IEEE Trans. Commun. 1995, 43, 158-162.

5. Cover, T.M.; Thomas, J.A. Elements of Information Theory, 2nd Ed.; Wiley: New York, NY, USA, 2006.

6. Yekhanin, S. Improved upper bound for the redundancy of fix-free codes. IEEE Trans. Inf. Theory 2004, 50, 2815-2818. 
7. Ahlswede, R.; Balkenhol, B.; Khachatrian, L. Some properties of fix-free codes. In Proceedings of International Seminar on Coding Theory and Combinatorics, Thahkadzor, Armenia, 6-11 October 1996; pp. 20-33.

8. Harada, K.; Kobayashi, K. A note on the fix-free code property. IEICE Trans. Fund. 1999, E82-A, 2121-2128.

9. Sergey, Y. Sufficient conditions of existence of fix-free codes. In Proceedings of IEEE International Symposium on Information Theory, Washington, DC, USA, 24-29 June 2001; p. 284.

10. Ye, C.; Yeung, R.W. Some basic properties of fix-free codes. IEEE Trans. Inf. Theory 2001, 47, $72-87$.

11. Kukorelly. Z.; Zeger, K. Sufficient conditions for existence of binary fix-free codes. IEEE Trans. Inf. Theory 2005, 51, 3433-3444.

12. Huffman, D.A. A method for the construction of minimum-redundancy codes. In Proceedings of I.R.E. September 1952; Volume 40, pp. 1098-1101.

13. Huang, Y.M.; Wu, T.Y.; Han, Y.S. An $A^{*}$-based algorithm for constructing reversible variable length codes with minimum average codeword length. IEEE Trans. Commun. 2010, 58, 3175-3185.

14. Savari, S.A.; Hossein Tabatabaei Yazdi, S.M.; Abedini, N.; Khatri, S.P. On optimal and achievable fix-free codes. IEEE Trans. Inf. Theory. 2012, 58, 5112-5129.

15. Esmaeili, M.; Kakhbod, A. On antiuniform and partially antiuniform sources. In Proceedings of IEEE International Conference on Communications, Istanbul, Turkey, 11-15 June 2006; pp. 1611-1615.

16. Esmaeili, M.; Kakhbod, A. On information theory parameters of infinite anti-uniform sources. IET Commun. 2007, 1, 1039-1041.

17. Humblet, P. Optimal source coding for a class of integer alphabets. IEEE Trans. Inf. Theory 1978, 24, 110-112.

18. Kato, A.; Han, T.; Nagaoka, H. Huffman coding with an infinite alphabet. IEEE Trans. Inf. Theory 1996, 42, 977-984.

19. Tsai, C.-W.; Wu, J.-L. Modified symmetrical reversible variable-length code and its theoretical bounds. IEEE Trans. Inf. Theory 2001, 47, 2543-2548.

20. Aghajan, A.; Khosravifard, M. Weakly symmetric fix-free codes. IEEE Trans. Inf. Theory 2014, 60, 5500-5515.

21. Fraenkel, A.S.; Klein, S.T. Bidirectional Huffman coding. Comput. J. 1990, 33, 296-307.

(c) 2015 by the authors; licensee MDPI, Basel, Switzerland. This article is an open access article distributed under the terms and conditions of the Creative Commons Attribution license (http://creativecommons.org/licenses/by/4.0/). 